\title{
Modifiable and non-modifiable risk factors for obstetric anal sphincter injury in a Norwegian Region: A case-control study.
}

\author{
Ragnhild Klokk ${ }^{1}$, Kjersti Bakken ${ }^{1}$, Trond Markestad ${ }^{2}$, and Mads Holten-Andersen ${ }^{1}$ \\ ${ }^{1}$ Innlandet Hospital Trust \\ ${ }^{2}$ University of Bergen
}

April 13, 2021

\begin{abstract}
Objective To identify modifiable and non-modifiable risk factors for severe obstetric anal sphincter injury (OASI) following vaginal delivery. Design Retrospective case-control study. Setting Single center maternity clinic in South-Eastern Norway Population Women diagnosed with OASI following singleton vaginal birth after 30 weeks' gestation $(\mathrm{n}=421)$ and matched controls $(n=421)$ during 1990-2002. Methods Data were extracted retrospectively from an institutional birth registry. For each woman with OASI the first subsequent vaginal singleton delivery matched for parity was elected as control. Potential determinants for OASI were assessed by conditional logistic regression analyses. Main outcome measure OASI, defined as 3rd or 4th degree obstetric anal sphincter lesions. Results Among modifiable factors amniotomy was the strongest independent determinant for OASI in both primi- (adjusted odds ratio [aOR] 4.84; 95\% CI 2.60-9.02) and multiparous (aOR 3.76; 95\% CI 1.45-9.76) women, followed by augmentation with oxytocin (primiparous: aOR 1.63; 95\% CI 1.08-2.46, multiparous: aOR 3.70; 95\% CI 1.79-7.67). Vacuum extraction and forceps delivery were independently associated with OASI in primiparous women (vacuum: aOR 1.91; 95\% CI 1.03-3.57, forceps: aOR 2.37; 95\% CI 1.14-4.92), and episiotomy for OASI in multiparous women (aOR 2.64; 95\% CI 1.36-5.14). Conclusions Amniotomy may be a hitherto unrecognized independent modifiable risk factor for OASI and should be further investigated for its potential role in preventive strategies for OASI. Funding Innlandet Hospital Trust research fund, grant number 150434. Keywords Obstetric anal sphincter injury; OASI; Birth; Birth injury; Modifiable risk factor; Amniotomy.
\end{abstract}

Modifiable and non-modifiable risk factors for obstetric anal sphincter injury in a Norwegian Region: A case-control study.

Ragnhild Klokk ${ }^{1}$, Kjersti S Bakken ${ }^{2,3}$, Trond Markestad ${ }^{4}$, Mads N Holten-Andersen ${ }^{1,5}$

${ }^{1}$ Department of Pediatrics, Innlandet Hospital Trust, Lillehammer, Norway

${ }^{2}$ Women's Clinic, Innlandet Hospital Trust, Lillehammer, Norway

${ }^{3}$ Centre for International Health, University of Bergen, Bergen, Norway

${ }^{4}$ Department of Research, Innlandet Hospital Trust, Brumunddal, Norway

${ }^{5}$ Institute of Clinical Medicine, Faculty of Medicine, University of Oslo, Oslo, Norway

\section{Corresponding author:}

Mads N Holten-Andersen, Department of Pediatrics, Innlandet Hospital Trust, Anders Sandviksgate, 2619 Lillehammer, Norway. 
Email: madsn.holten-andersen@sykehuset-innlandet.no

Telephone: +4741487829

Running title:

Risk factors for anal sphincter injury

Abstract

Objective To identify modifiable and non-modifiable risk factors for severe obstetric anal sphincter injury (OASI) following vaginal delivery.

Design Retrospective case-control study.

Setting Single center maternity clinic in South-Eastern Norway

Population Women diagnosed with OASI following singleton vaginal birth after 30 weeks' gestation ( $\mathrm{n}=$ 421) and matched controls $(\mathrm{n}=421)$ during 1990-2002.

Methods Data were extracted retrospectively from an institutional birth registry. For each woman with OASI the first subsequent vaginal singleton delivery matched for parity was elected as control. Potential determinants for OASI were assessed by conditional logistic regression analyses.

Main outcome measure OASI, defined as $3^{\text {rd }}$ or $4^{\text {th }}$ degree obstetric anal sphincter lesions.

Results Among modifiable factors amniotomy was the strongest independent determinant for OASI in both primi- (adjusted odds ratio [aOR] 4.84; 95\% CI 2.60-9.02) and multiparous (aOR 3.76; 95\% CI 1.45-9.76) women, followed by augmentation with oxytocin (primiparous: aOR 1.63; 95\% CI 1.08-2.46, multiparous: aOR 3.70; 95\% CI 1.79-7.67). Vacuum extraction and forceps delivery were independently associated with OASI in primiparous women (vacuum: aOR 1.91; 95\% CI 1.03-3.57, forceps: aOR 2.37; 95\% CI 1.14-4.92), and episiotomy for OASI in multiparous women (aOR 2.64; 95\% CI 1.36-5.14).

Conclusions Amniotomy may be a hitherto unrecognized independent modifiable risk factor for OASI and should be further investigated for its potential role in preventive strategies for OASI.

Funding Innlandet Hospital Trust research fund, grant number 150434.

Keywords Obstetric anal sphincter injury; OASI; Birth; Birth injury; Modifiable risk factor; Amniotomy.

\section{Introduction}

Most women experience perineal trauma when giving birth (1). Severe perineal lesions, referred to as obstetric anal sphincter injury (OASI), are diagnosed in as many as $11 \%$ of vaginal deliveries, but with significant variation between studies and national birth statistics (1-5). The true incidence rate for severe lesions may be in the range of 10-26\% because the injuries can be overlooked at the delivery wards or be occult $(4,6$, 7). Apart from the immediate perineal pain, OASI often has short- and long-term consequences including negative impact on sexual life and quality of life in general (8-12) as well as anal incontinence (11-15).

Adequate clinical examination following delivery is pivotal in the diagnosis of OASI (6, 16-19), and increased awareness and training of health care personnel have resulted in a doubling of detection rates $(2,18,19)$. Alongside the focus on detection, prevention has gained increasing attention. Obstetric training programs for midwives with emphasis on potential preventive measures, such as attention to maternal birth position and perineal massage during the second stage of labour, have been suggested as ways of decreasing the risk of OASI (20-22). Implementation of a preventive program in five maternity clinics in Norway resulted in decreased prevalence of OASI (23), as has similar programs in other European countries (14, 24-27). However, the evidence of persistent efficacy of preventive programs is low, partly because the existing studies were assessed shortly after their introduction (28). In a study involving the four large Nordic countries over seven years, a lasting reduction was only observed in Norway (29). 
Established risk factors for OASI include primiparity, vaginal birth after caesarean delivery, advanced maternal age, high birthweight, fetal occiput posterior presentation, induction and augmentation of labour, instrumental delivery, increased duration of second stage of labour, episiotomy, and Asian ethnicity (1, 2, 22, 30-32). A recent meta-analysis showed that the incidence of OASI remains high and it highlighted a need to search for hitherto unrecognized and potentially modifiable risk factors (1). We aimed at exploring both modifiable and non-modifiable risk factors in a large retrospective case-control study based on a regional cohort where detailed information related to maternal, pregnancy, delivery, and fetal characteristics had been collected prospectively.

\section{Methods}

\section{Participants}

At Innlandet Hospital Trust, Lillehammer, Norway, detailed information on maternal health, pregnancy, delivery and the postpartum period until discharge is prospectively registered in a perinatal database. This hospital covers virtually all births in a region with a population of around 90,000 people at the time of the study; around 23,000 lived in the city Lillehammer and the others in rural areas with small towns. The women were generally first registered in the perinatal database at 18-20 weeks' gestation when they met for the routine ultrasound assessment. This study included all deliveries that occurred during January $1^{\text {st }}$ 1990 to December $31^{\text {st }} 2002$. We identified singleton vaginal deliveries with gestational age $>210$ days where women for the first time were diagnosed with perineal rupture from the database, and the data were quality assured and expanded by scrutinizing delivery protocols, charts, and patient records. Women with $3^{\text {rd }}$ and $4^{\text {th }}$ degree OASI were defined as cases, and we selected the next vaginal singleton delivery with the same parity and gestational age $>210$ days without OASI as a matched control.

\section{Definitions and interventions}

OASI was diagnosed according to the International Classification of Diseases (ICD) 9 definition 664.2 and 664.3 (similar to ICD 10 codes O70.2 and O70.3). The diagnosis was set at the time of the tear by the midwife or physician in charge of the delivery and subsequently confirmed by a specialist in obstetrics and gynecology.

In addition to degree of perineal rupture, modifiable and non-modifiable variables regarding the infant, mother and birth process were registered. Non-modifiable variables included birth weight (in grams), length (in $\mathrm{cm}$ ), head circumference (in $\mathrm{cm}$ ), gestational age (in days), and Apgar score at 1 and 5 minutes postpartum of the newborn, and maternal age (in years), parity, duration of the first and second stage of labour (in minutes), and fetal presentation (occiput posterior, occiput anterior, deep transverse, breech). Modifiable variables included the mother's birth position (supine/sitting, side bearing, standing, kneeling, and on stool), induction of labour (yes/no), amniotomy (yes/no), episiotomy (mediolateral, yes/no), augmentation with oxytocin (yes/no), application of fundal pressure (yes/no), and instrumental delivery by vacuum extraction (yes/no) or forceps (yes/no).

Methods used for induction of labour were based on the Bishop scores and included membrane sweeping, transcervical Foley catheter, prostaglandin vaginal tablets, amniotomy or/and augmentation with oxytocin. Amniotomy was performed in births with a spontaneous onset to shorten duration of the first and second stage of labour or when continuous surveillance of the fetus with a scalp-electrode or an examination of the amniotic fluid was considered necessary. Augmentation with oxytocin was used after amniotomy in cases of labour dystocia in births with spontaneous onset. Indications for performing an episiotomy included imminent fetal asphyxia, preterm birth, and instrumental vaginal delivery which included vacuum extraction and the use of forceps at the physician's discretion.

Birth weight was measured and registered in grams and subsequently categorized into quartiles: $<3300$, 3300-3650, 3660-4040, and [?]4040 grams. Crown-heel length and head circumference were measured in centimeters according to protocol. Gestational age was estimated according to routine ultrasonography at 18-20 weeks of gestation at Lillehammer Hospital. Apgar scores were assessed at one and five minutes 
following birth by midwives or physicians. The respective scores were subsequently dichotomized into $<7$ or [?]7. Maternal age was registered in years and subsequently categorized into the following three groups: $<25$, 25-29, and [?]30 years. The cases and controls were stratified to primiparous (first birth) or multiparous ([?]second birth).

\section{Statistics}

Missing data were treated by listwise deletion. Continuous variables were tested for distributions of normality and described by means and standard deviations. Categorical variables were described by frequencies and proportions. We performed separate analyses for the primi- and multiparous pregnancies. For the variables within each group, we analyzed differences between cases and controls with t-tests, Kruskal-Wallis or $\mathrm{Chi}^{2}{ }_{-}$ tests. Significance level was set at $5 \%$. Correlation matrices demonstrated covariation between weight, length and head circumference of the infant, and only birthweight was used in logistic regression analyses. We used univariate conditional logistic regression analyses when assessing associations between exposure variables and OASI. Subsequently, we built risk-factor models for OASI by using multivariate conditional logistic regression analyses progressing with a stepwise procedure. In this analysis we included variables that were significantly different between the cases and controls in the univariate analyses. We assessed multicollinearity by using variance inflation factor (VIF). We assessed interactions between amniotomy and the following variables: augmentation with oxytocin, episiotomy, and instrumental delivery by vacuum or forceps. STATA 15.1 software (STATA, College Station, TX, United States: StataCorp, 2017) was used for all the analyses.

\section{Ethics}

The research project was approved by the Norwegian Social Science Data Services (project number: 2614) and The Norwegian Data Protection Authority (reference code: 95/2691-2 GS $\varnothing$ ).

\section{Funding}

The study was funded by Innlandet Hospital Trust research fund under grant number 150434 .

\section{Results}

During this 13-year period, 12,883 women gave birth at the study hospital, and 11,374 of them had a vaginal delivery. The mean incidence of OASI was $2.9 \%$ but increased gradually from $2.4 \%$ in 1990 to a maximum of $5.9 \%$ in 2002 . Following vaginal delivery, 421 women $(3.7 \%)$ were diagnosed with first time $3^{\text {rd }}(\mathrm{n}=324)$ or $4^{\text {th }}$ degree $(\mathrm{n}=97)$ OASI and included in the study along with 421 matched controls without OASI. Of the 842 women, $550(65.3 \%)$ were primiparous and $292(34.7 \%)$ multiparous. The median parity of the multiparous women was the second child, and the highest parity was six.

Of the non-modifiable variables, we found that maternal age, infant weight, length, head circumference and gestational age, and duration of both the first and second stage of labour were significantly higher for both the primi- and multiparous women with OASI compared to their respective controls (Table 1). Of the potentially modifiable variables related to the delivery, we found that frequencies of episiotomy, amniotomy, augmentation with oxytocin, supine or sitting birth position, instrumental delivery with vacuum extraction and forceps were significantly higher for both the primi- and multiparous women with OASIS compared to their controls (Table 2). Induction of labour occurred more often in the primiparous women with OASI (Table 2).

In the final multivariate conditional logistic regression model, higher maternal age and birthweight for primiparous women and birthweight for the multiparous women were the only non-modifiable variables associated with rates of OASI (Tables 3 and 4). Of the modifiable variables, amniotomy was strongly associated with OASI, both in the primiparous (OR 4.84, 95\% CI 2.60-9,02) and multiparous (OR 3.76, 95\% CI 1.45-9.76) women, as was augmentation with oxytocin (OR 1.63, 95\% CI 1.08-2.46 and 3.70, 95\% CI 1.79-7.67, respectively, Tables 3 and 4). Instrumental delivery was associated with OASI in the primiparous women (Table 
3) and episiotomy with OASI in the multiparous women (Table 4). We found no significant interactions or multicollinearities.

\section{Discussion}

In this unselected population, OASI was associated with known non-modifiable factors like high maternal age, first pregnancy, and large babies. Of potentially modifiable factors, OASI was associated with induction of labour and instrumental vaginal delivery in primiparous women, and with amniotomy and augmentation with oxytocin in both primi- and multiparous women, procedures that are primarily initiated to accelerate delivery.

The major strengths of this study were the unselected population, the large number of participants, and completeness of data. We also consider the inclusion of only one obstetric hospital a strength since no major official changes in routines were introduced, although we cannot exclude gradual unrecognized changes during this 13-year period. The retrospective nature of the study may be a weakness since reasons for performing amniotomy and augmentation with oxytocin were not necessarily specified and since vigilance in classifying perineal rupture may have been less accurate than in a planned prospective study. Furthermore, the women were not routinely followed after discharge from the hospital. The time lap between the collection and publication of data may make the results less valid of today's practice since increased focus on reducing the incidence of OASI has been implemented since the data were collected (20,23). However, the use of induction of labour and augmentation with oxytocin have increased nationally since these data were collected, and the risks related to these interventions may correspond to what we found (33).

To our knowledge, our study is the first to include amniotomy as a potential independent risk factor for OASI. Amniotomy was the strongest independent modifiable risk factor regardless of parity and suggests that attention to indications and timing of amniotomy may be a hitherto unrecognized means of preventing OASI. The use of amniotomy varies between institutions both in Norway and other countries and ranges from $20 \%$ to $60 \%(34,35)$. However, in our experience the documentation of amniotomy in patient charts during labour is highly variable. Even though we have a national high-quality birth register in Norway, the use of amniotomy in spontaneous labour is not reported (33).

With the goal of reducing cesarean births through active management of labour, amniotomy has been widely and readily accepted to avoid labour for more than 12 hours (36). However, reducing length of labour might not be a benefit for all women, and a Cochrane review from 2013 concluded that there is no evidence to support routine amniotomy to shorten spontaneous labour or to avoid prolonged labour (37). The mechanism behind the association between amniotomy and OASI is unclear, but we speculate that amniotomy may disrupt the normal physiologic process of gradual adaptation of the birth canal and thereby a higher risk of trauma.

In the present study, we also found that augmentation with oxytocin was an independent risk factor for OASI for both primi- and multiparous women. This is in accordance with previous studies $(1,38)$. Augmentation with oxytocin is widely used when labour is delayed, and probably more than half of women in labour worldwide receive oxytocin augmentation $(33,35,39)$. However, this varies widely between countries and within the same country. In our study, $60 \%$ of the primiparous and $46.7 \%$ of the multiparous women were augmented with oxytocin, which is in line with current rates in maternity wards in Norway (35). Increased and reduced control of contractions are known potential adverse effect of augmentation of labour with oxytocin (40). We suggest that the effects of augmentation with oxytocin are similar to that of amniotomy in that the birth progress may be more rapid than the natural adaptation of the birth canal.

Instrumental vaginal delivery is a well-established risk factor for OASI $(1,2,6,31)$. However, this was only an independent risk factor for primiparous women in our study. Instrumental delivery was also associated with OASI in multiparous women in the unadjusted analysis, and the reason for no significant association in the adjusted analysis may partly be that the study lacked power to detect a risk since instruments were rarely used in this group. 
In conclusion, the study suggests that indications for and timing of amniotomy and augmentation of the birth process with oxytocin need to be readdressed in order to reduce the risk of severe perineal ruptures.

\section{Disclosure of interests:}

No conflict of interest for any of the authors.

\section{Contribution to Authorship:}

As the principal investigator, RK contributed in the conceptualization, planning and carrying out of the study. RK also participated in the analyses of the data and writing of the manuscript.

KSB contributed in the analyses of the data and the writing of the manuscript.

TM contributed in the conceptualization, planning and carrying out of the study and in the writing of the manuscript. TM also supervised the analyses of the data

MNH-A carried out the washing and analyses of the data and contributed in the writing of the manuscript.

The authors accept responsibility for the paper as published.

\section{Details of Ethics Approval:}

The research project was approved by the Norwegian Social Science Data Services (project number: 2614) and The Norwegian Data Protection Authority (reference code: 95/2691-2 GSØ).

\section{References}

1. Pergialiotis V, Bellos I, Fanaki M, Vrachnis N, Doumouchtsis SK. Risk factors for severe perineal trauma during childbirth: An updated meta-analysis. Eur J Obstet Gynecol Reprod Biol. 2020;247:94-100.

2. Ampt AJ, Ford JB, Roberts CL, Morris JM. Trends in obstetric anal sphincter injuries and associated risk factors for vaginal singleton term births in New South Wales 2001-2009. Aust N Z J Obstet Gynaecol. 2013;53(1):9-16.

3. Jha S, Parker V. Risk factors for recurrent obstetric anal sphincter injury (rOASI): a systematic review and meta-analysis. Int Urogynecol J. 2016;27(6):849-57.

4. Dudding TC, Vaizey CJ, Kamm MA. Obstetric anal sphincter injury: incidence, risk factors, and management. Ann Surg. 2008;247(2):224-37.

5. Laine K, Gissler M, Pirhonen J. Changing incidence of anal sphincter tears in four Nordic countries through the last decades. Eur J Obstet Gynecol Reprod Biol. 2009;146(1):71-5.

6. Sultan AH, Kamm MA, Hudson CN, Thomas JM, Bartram CI. Anal-sphincter disruption during vaginal delivery. N Engl J Med. 1993;329(26):1905-11.

7. Oberwalder M, Connor J, Wexner SD. Meta-analysis to determine the incidence of obstetric anal sphincter damage. Br J Surg. 2003;90(11):1333-7.

8. Gommesen D, Nohr E, Qvist N, Rasch V. Obstetric perineal tears, sexual function and dyspareunia among primiparous women 12 months postpartum: a prospective cohort study. BMJ Open. 2019;9(12):e032368.

9. Evers EC, Blomquist JL, McDermott KC, Handa VL. Obstetrical anal sphincter laceration and anal incontinence 5-10 years after childbirth. Am J Obstet Gynecol. 2012;207(5):425 e1-6.

10. Cornelisse S, Arendsen LP, van Kuijk SM, Kluivers KB, van Dillen J, Weemhoff M. Obstetric anal sphincter injury: a follow-up questionnaire study on longer-term outcomes. Int Urogynecol J. 2016;27(10):1591-6.

11. Pollack J, Nordenstam J, Brismar S, Lopez A, Altman D, Zetterstrom J. Anal incontinence after vaginal delivery: a five-year prospective cohort study. Obstet Gynecol. 2004;104(6):1397-402. 
12. Wagenius J, Laurin J. Clinical symptoms after anal sphincter rupture: a retrospective study. Acta Obstet Gynecol Scand. 2003;82(3):246-50.

13. Gommesen D, Nohr EA, Qvist N, Rasch V. Obstetric perineal ruptures-risk of anal incontinence among primiparous women 12 months postpartum: a prospective cohort study. Am J Obstet Gynecol. 2020;222(2):165 e1- e11.

14. Laine K, Skjeldestad FE, Sandvik L, Staff AC. Prevalence and Risk Indicators for Anal Incontinence among Pregnant Women. ISRN Obstet Gynecol. 2013;2013:947572.

15. Leeman L, Rogers R, Borders N, Teaf D, Qualls C. The Effect of Perineal Lacerations on Pelvic Floor Function and Anatomy at 6 Months Postpartum in a Prospective Cohort of Nulliparous Women. Birth. 2016;43(4):293-302.

16. Groom KM, Paterson-Brown S. Can we improve on the diagnosis of third degree tears? Eur J Obstet Gynecol Reprod Biol. 2002;101(1):19-21.

17. Andrews V, Sultan AH, Thakar R, Jones PW. Risk factors for obstetric anal sphincter injury: a prospective study. Birth. 2006;33(2):117-22.

18. Andrews V, Thakar R, Sultan AH. Structured hands-on training in repair of obstetric anal sphincter injuries (OASIS): an audit of clinical practice. Int Urogynecol J Pelvic Floor Dysfunct. 2009;20(2):193-9.

19. Harvey MA, Pierce M, Alter JE, Chou Q, Diamond P, Epp A, et al. Obstetrical Anal Sphincter Injuries (OASIS): Prevention, Recognition, and Repair. J Obstet Gynaecol Can. 2015;37(12):1131-48.

20. Laine K, Skjeldestad FE, Sandvik L, Staff AC. Incidence of obstetric anal sphincter injuries after training to protect the perineum: cohort study. BMJ Open. 2012;2(5).

21. Naidu M, Sultan AH, Thakar R. Reducing obstetric anal sphincter injuries using perineal support: our preliminary experience. Int Urogynecol J. 2017;28(3):381-9.

22. Tunestveit JW, Baghestan E, Natvig GK, Eide GE, Nilsen ABV. Factors associated with obstetric anal sphincter injuries in midwife-led birth: A cross sectional study. Midwifery. 2018;62:264-72.

23. Hals E, Oian P, Pirhonen T, Gissler M, Hjelle S, Nilsen EB, et al. A multicenter interventional program to reduce the incidence of anal sphincter tears. Obstet Gynecol. 2010;116(4):901-8.

24. De Meutter L, A DvH, van der Woerdt-Eltink I, de Leeuw JW. Implementation of a perineal support programme for reduction of the incidence of obstetric anal sphincter injuries and the effect of non-compliance. Eur J Obstet Gynecol Reprod Biol. 2018;230:119-23.

25. Skriver-Moller AC, Madsen ML, Poulsen MO, Overgaard C. Do we know enough? A quality assessment of the Finnish intervention to prevent obstetric anal sphincter injuries. J Matern Fetal Neonatal Med. 2016;29(21):3461-6.

26. Jango H, Westergaard HB, Kjaerbye-Thygesen A, Langhoff-Roos J, Lauenborg J. Changing incidence of obstetric anal sphincter injuries-A result of formal prevention programs? Acta Obstet Gynecol Scand. 2019;98(11):1455-63.

27. Gurol-Urganci I, Bidwell P, Sevdalis N, Silverton L, Novis V, Freeman R, et al. Impact of a quality improvement project to reduce the rate of obstetric anal sphincter injury: a multicentre study with a steppedwedge design. BJOG. 2021;128(3):584-92.

28. Poulsen MO, Madsen ML, Skriver-Moller AC, Overgaard C. Does the Finnish intervention prevent obstetric anal sphincter injuries? A systematic review of the literature. BMJ Open. 2015;5(9):e008346.

29. Laine K, Rotvold W, Staff AC. Are obstetric anal sphincter ruptures preventable?- large and consistent rupture rate variations between the Nordic countries and between delivery units in Norway. Acta Obstet Gynecol Scand. 2013;92(1):94-100. 
30. Hehir MP, Fitzpatrick M, Cassidy M, Murphy M, O'Herlihy C. Are women having a vaginal birth after a previous caesarean delivery at increased risk of anal sphincter injury? BJOG. 2014;121(12):1515-20.

31. Ramm O, Woo VG, Hung YY, Chen HC, Ritterman Weintraub ML. Risk Factors for the Development of Obstetric Anal Sphincter Injuries in Modern Obstetric Practice. Obstet Gynecol. 2018;131(2):290-6.

32. Selmer-Olsen T, Nohr EA, Tappert C, Eggebo TM. Incidence and risk factors for obstetric anal sphincter ruptures, OASIS, following the introduction of preventive interventions. A retrospective cohort study from a Norwegian hospital 2012-2017. Sex Reprod Healthc. 2019;22:100460.

33. The Norwegian Institute of Public Health. 2021 [cited 2021 24.03.2021]. Available from: https://www.fhi.no/en/hn/health-registries/medical-birth-registry-of-norway/.

34. Seijmonsbergen-Schermers AE, Zondag DC, Nieuwenhuijze M, van den Akker T, Verhoeven CJ, Geerts $\mathrm{CC}$, et al. Regional variations in childbirth interventions and their correlations with adverse outcomes, birthplace and care provider: A nationwide explorative study. PLoS One. 2020;15(3):e229488.

35. Gaudernack LC, Froslie KF, Michelsen TM, Voldner N, Lukasse M. De-medicalization of birth by reducing the use of oxytocin for augmentation among first-time mothers - a prospective intervention study. BMC Pregnancy Childbirth. 2018;18(1):76.

36. O’Driscoll K MD, Boylan P. Active management of labour. 3rd ed. London: Mosby; 1993.

37. Smyth RM, Alldred SK, Markham C. Amniotomy for shortening spontaneous labour. Cochrane Database Syst Rev. 2013(1):CD006167.

39. Rygh AB, Skjeldestad FE, Korner H, Eggebo TM. Assessing the association of oxytocin augmentation with obstetric anal sphincter injury in nulliparous women: a population-based, case-control study. BMJ Open. 2014;4(7):e004592.

39. Zhang J, Branch DW, Ramirez MM, Laughon SK, Reddy U, Hoffman M, et al. Oxytocin regimen for labor augmentation, labor progression, and perinatal outcomes. Obstet Gynecol. 2011;118(2 Pt 1):249-56.

40. Oscarsson ME, Amer-Wahlin I, Rydhstroem H, Kallen K. Outcome in obstetric care related to oxytocin use. A population-based study. Acta Obstet Gynecol Scand. 2006;85(9):1094-8.

\section{Hosted file}

Tables_050421.pdf available at https://authorea.com/users/407526/articles/517815-modifiableand-non-modifiable-risk-factors-for-obstetric-anal-sphincter-injury-in-a-norwegianregion-a-case-control-study 